\title{
Inverse Mathematical Models for Brain Tumour Growth
}

\author{
Rym Jaroudi
}

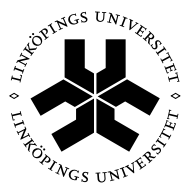

Linköping University

INSTITUTE OF TECHNOLOGY 
Inverse Mathematical Models for Brain Tumour Growth

Rym Jaroudi

LIU-TEK-LIC 2017

ISBN 978-91-7685-440-2

ISSN 0280-7971

Linköping University

Department of Science and Technology

SE-601 74 Norrköping

Printed by LiU Tryck, Linköping, Sweden 2017 


\section{Abstract}

We study the following well-established model of reaction-diffusion type for brain tumour growth:

$$
\left\{\begin{array}{rlrl}
\partial_{t} u-\operatorname{div}(D(x) \nabla u)-f(u) & =0, & \text { in } \Omega \times(0, T) \\
u(0) & =\varphi, & \text { in } \Omega \\
D \nabla u \cdot n & =0, & & \text { on } \partial \Omega \times(0, T)
\end{array}\right.
$$

This equation describes the change over time of the normalised tumour cell density $u$ as a consequence of two biological phenomena: proliferation and diffusion.

We discuss a mathematical method for the inverse problem of locating the brain tumour source (origin) based on the reaction-diffusion model. Our approach consists in recovering the initial spatial distribution of the tumour cells $\varphi=u(0)$ starting from a later state $\psi=u(T)$, which can be given by a medical image. We use the nonlinear Landweber regularization method to solve the inverse problem as a sequence of well-posed forward problems.

We give full 3-dimensional simulations of the tumour in time on two types of data, the 3d Shepp-Logan phantom and an MRI T1weighted brain scan from the Internet Brain Segmentation Repository (IBSR). These simulations are obtained using standard finite difference discretisation of the space and time-derivatives, generating a simplistic approach that performs well. We also give a variational formulation for the model to open the possibility of alternative derivations and modifications of the model. Simulations with synthetic images show the accuracy of our approach for locating brain tumour sources. 



\section{Acknowledgments}

I would like to warmly thank my supervisor George Baravdish for his guidance and support during the last two years. He was always present to help and motivate me with his deep knowledge, unshakable enthusiasm, and strong passion. I thank him for his help and vision, and accepting me within the team, where he created a friendly research atmosphere.

I am extremely grateful to my co-supervisor B. Tomas Johansson for valuable discussions and for helpful suggestions during this work. I thank him for his excellent guidance, careful paper reading, and encouragement. I really appreciated our numerous meetings and their inputs which were necessary to complete this thesis.

I also thank Freddie Åström for his assistance with the numerical experiments presented in the thesis. I thank him for the time and work he put in this thesis which were essential to shape it.

I want to express my gratitude for the Alyssa program grant from Erasmus Mundus Action 2, Lot 6 EU funding for giving me this wonderful opportunity to experience living in Sweden making this work possible.

My sincere thanks to the Department of Science and Technology at Campus Norrköping, Linköping University for providing an excellent work opportunity within great research environment.

Last but not least, I want to thank my family and friends whom were always there for me. Coming back to Tunis was always motivating.

Norrköping, September 2017

Rym 



\section{Contents}

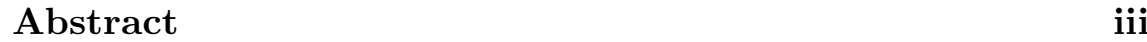

Acknowledgments $\quad$ v

1 Introduction 1

1.1 Outline of the thesis $\ldots \ldots \ldots \ldots$

2 Mathematical Preliminaries 5

2.1 Function spaces . . . . . . . . . . . . 5

2.2 Ill-Posed and Inverse Problems . . . . . . . . . . . 7

2.3 Iterative regularization methods . . . . . . . . . . . 10

3 Mathematical Models for Brain Tumours $\quad 19$

3.1 Brain Tumours . . . . . . . . . . . . . . . . . . 19

3.2 Reaction-Diffusion Models . . . . . . . . . . . . . . 23

3.3 Discretization . . . . . . . . . . . . . . . 28

$\begin{array}{ll}\text { Bibliography } & 31\end{array}$

4 Summary of Papers $\quad 37$

4.1 Paper 1: Source Localization of Reaction-Diffusion Models for Brain Tumors . . . . . . . . . . . . . . . . 37

4.2 Paper 2: Numerical simulations in 3-dimensions of reactiondiffusion models for brain tumours growth . . . . . . . 38

4.3 Paper 3: Numerical reconstruction of brain tumours . 38

$\begin{array}{ll}\text { Paper } 1 & 41\end{array}$

$\begin{array}{ll}\text { Paper 2 } & 55\end{array}$

$\begin{array}{ll}\text { Paper } 3 & \mathbf{7 7}\end{array}$ 
Contents

viii 


\section{Chapter 1}

\section{Introduction}

Brain cancer is still one of the most common cancers worldwide. A powerful tool in understanding cancer development is the use of mathematical models and simulations for determining the future state of the tumour by prediction or its initial state by source localization. Popular models for brain tumour growth are based on partial differential equations (PDEs) of reaction-diffusion type:

$$
\left\{\begin{aligned}
\partial_{t} u-\operatorname{div}(D(x) \nabla u)-f(u) & =0, & \text { in } \Omega \times(0, T) \\
u(0) & =\varphi, & \text { in } \Omega \\
D \nabla u \cdot n & =0, & \text { on } \partial \Omega \times(0, T)
\end{aligned}\right.
$$

with $u=u(x, t)$ the normalised tumour cell density at a time $t$ in a position $x$ of the brain region $\Omega$. The diffusion of the tumour cells is in the term $\operatorname{div}(D(x) \nabla u)$ and their proliferation is modelled by $f(u)$. The research frontier moves quickly in applied mathematics of cancer research and ever more complex models are proposed for better forecasting of the growth of tumours. For newcomers to the field, it can be beneficial to see simulations of standard basic models to get a feeling for their behaviour and shortcomings. To our best knowledge, only two-dimensional simulations seem presented in the literature for this type of models. Since the models are inevitably non-linear the simplification to 2-dimensions could render different and spurious behaviour. Moreover, despite the large potential impact of tumour source localization, few works addressed this problem. Thus, in this thesis, we undertake the laborious task of doing full 3-dimensional simulations on two problems: the direct problem and the inverse problem for brain tumours, while keeping our focus on the source localization problem, since it is less studied in the litera- 
ture. We start by studying the direct problem, we show how existence and uniqueness of a solution to the reaction-diffusion model can be obtained using basic concepts of weak formulations. Furthermore, we also present a variational formulation that can aid in deriving and modifying the reaction-diffusion model. Then, we approach the problem of reconstructing the source of cell densities evolved within the reaction-diffusion model commonly used to describe brain tumour growth. That is, recovering the initial spatial distribution of the tumour cells $\varphi=u(0)$ starting from a later state $\psi=u(T)$ :

$$
\left\{\begin{aligned}
\partial_{t} u-\operatorname{div}(D(x) \nabla u)-f(u) & =0, & \text { in } \Omega \times(0, T) \\
u(T) & =\psi, & \text { in } \Omega \\
D \nabla u \cdot n & =0, & \text { on } \partial \Omega \times(0, T)
\end{aligned}\right.
$$

This inverse problem of solving the reaction diffusion model (1.1) backwards in time is an ill-posed problem in the sense of Hadamard [1]. Unlike the majority of research done in the area of tumour source localization, which aim at approximating the non-linear PDE models by systems of ordinary differential equations ODEs (for example with the Eikonal equation), we proposed a novel and altogether different mathematical approach taking advantage of the theory of inverse problems to reconstruct initial data in parabolic equations, that is we recast the reconstruction of the source as a backward parabolic problem. In doing this, we remove sources of uncertainty introduced when reducing the model to ODEs, i.e., we work with the reactiondiffusion model directly. Although backward parabolic problems are classical from a theoretical point of view, they still have to be carefully regularized to obtain a stable numerical solution. In the linear case, iterative regularizing methods that preserve the governing PDE have been successfully employed. This field was developed through ideas of V. A. Kozlov and V. G. Maz'ya [2] that treated the abstract parabolic equation with a self-adjoint operator coefficient acting on a Hilbert space and a final value condition. G. Baravdish [3] extended these iterative method to an abstract first order differential equation.

In this thesis, we extend some of the ideas of Kozlov, Maz'ya and Baravdish, to propose a non-linear Landweber method for solving the backward reaction diffusion equation (1.2). We present 3-dimensional numerical simulations showing that stable numerical solutions to the source reconstruction can be obtained. An initial study was first done for the 2-dimensional case, and we later build on this and extend into 3-dimensions. We did not use any commercial PDE solver but show 
instead how a rather straightforward finite difference scheme can be employed to render the simulations, based on work of F. Åström [4]. In the simulations done on a realistic set up, that is the standard 3-dimensional Shepp-Logan phantom and an MRI T1-weighted brain scan from the Internet Brain Segmentation Repository (IBSR) describing a more complex geometry, we use different parameters including non-linearities for the forward model compared with the backward model as a way to simulate noise in the data. The analysis and simulations altogether counts as the main novelty of the present work. It is not aimed to get perfectly modelled results; we use a standard widely used model but there are extensions of it for even better prediction of tumour growth. The current work can be seen as an initial start. With the developed techniques, one can then make simulations for backward problems for the more advanced tumour models.

\subsection{Outline of the thesis}

Chapter two presents an overview of the mathematical preliminaries necessary to understand the underlying theory of our approach. In particular, we present theory from partial differential equations, illposed problems and analysis related to the reaction-diffusion model. The third chapter reviews the biological aspects of brain tumours as well as alternative approaches to describe brain tumour growth. The fourth chapter contains a summary of the following published and submitted papers:

- Paper 1: Source Localization of Reaction-Diffusion Models for Brain Tumors

- Paper 2: Numerical simulations in 3-dimensions of reactiondiffusion models for brain tumours growth

- Paper 3: Numerical reconstruction of brain tumours 
Chapter 1. Introduction 


\section{Chapter 2}

\section{Mathematical Preliminaries}

In this chapter, we introduce some of the mathematical tools needed in the following chapters. These tools are from the theory of partial differential equations [5] and from the theory of ill-posed and inverse problems [6].

\subsection{Function spaces}

Let $\Omega$ be an open domain in $\mathbf{R}^{n}$. Let $X$ denote a real Banach space, with norm $\|\cdot\|$.

Definition 2.1.1. The space $L^{p}(0, T ; X)$ consists of all measurable functions $u:(0, T) \rightarrow X$ with

$$
\|u\|_{L^{p}(0, T ; X)}:=\left\{\begin{array}{cc}
\left(\int_{0}^{T}\|u(t)\|^{p} d t\right)^{1 / p}<\infty, & 1 \leq p<\infty, \\
{\operatorname{ess~} \sup _{0 \leq t \leq T}\|u(t)\|,}_{p=\infty .}
\end{array}\right.
$$

Definition 2.1.2. The space $C(0, T ; X)$ comprises of all continuous functions $u:(0, T) \rightarrow X$ with

$$
\|u\|_{C(0, T ; X)}:=\max _{0 \leq t \leq T}\|u(t)\|<\infty .
$$

Definition 2.1.3. Let $u \in L^{1}(0, T ; X)$. We say $v \in L^{1}(0, T ; X)$ is the weak derivative of $u$ written

$$
u^{\prime}=v,
$$


provided

$$
\int_{0}^{T} \phi^{\prime}(t) u(t) d t=-\int_{0}^{T} \phi(t) v(t) d t
$$

for all scalar test functions $\phi \in C_{c}^{\infty}(0, T)$ (the space of infinitely differentiable functions with compact support.)

Definition 2.1.4. The space $H^{m}(\Omega)$ where $m$ a positive integer, denotes the set of all functions $u$ defined in $\Omega$ such that its distributional derivatives of order $|s|=\sum_{i=1}^{m} s_{i} \leq m$ all belong to $L^{2}(\Omega)$. Furthermore, $H^{m}(\Omega)$ is a Hilbert space with the norm

$$
\|u\|_{H^{m}(\Omega)}=\left(\sum_{s \leq m} \int_{\Omega}\left|\frac{\partial^{s} u}{\partial x^{s}}\right|^{2} d x\right)^{1 / 2}
$$

Definition 2.1.5. The Sobolev space $W^{1, p}(0, T ; X)$ consists of all functions $u \in L^{p}(0, T ; X)$ such that $u^{\prime}$ exists in the weak sense and belongs to $L^{p}(0, T ; X)$. Furthermore,

$$
\|u\|_{W^{1, p}(0, T ; X)}:=\left\{\begin{array}{cc}
\left(\int_{0}^{T}\|u(t)\|^{p}+\left\|u^{\prime}(t)\right\|^{p} d t\right)^{1 / p}<\infty, & 1 \leq p<\infty \\
\operatorname{ess}^{1 / p} \sup _{0 \leq t \leq T}\left(\|u(t)\|+\left\|u^{\prime}(t)\right\|\right), & p=\infty .
\end{array}\right.
$$

For simple notations, we put $H^{1}(0, T ; X)=W^{1,2}(0, T ; X)$.

Theorem 2.1.6. Let $u \in W^{1, p}(0, T ; X)$ for some $1 \leq p \leq \infty$. Then

- $u \in C(0, T ; X)$.

- $u(t)=u(s)+\int_{s}^{t} u^{\prime}(\tau) d \tau, \quad 0 \leq s \leq t \leq T$.

- We have the estimate

$$
\max _{0 \leq t \leq T}\|u(t)\| \leq C\|u\|_{W^{1, p}(0, T ; X)}
$$

with the constant $C$ depending only on $T$.

In the case of $p=2$ and $X=H_{0}^{1}(\Omega)$, we have

- $u \in C\left(0, T ; L^{2}(\Omega)\right)$.

- The mapping $t \mapsto\|u(t)\|_{L^{2}(U)}^{2}$ is absolutely continuous with

$$
\frac{d}{d t}\|u(t)\|_{L^{2}(U)}^{2}=2\left\langle u^{\prime}(t), u(t)\right\rangle, \quad 0 \leq t \leq T .
$$


- We have the estimate

$$
\max _{0 \leq t \leq T}\|u(t)\|_{L^{2}(U)} \leq C\left(\|u\|_{L^{2}\left(0, T ; H_{0}^{1}(U)\right)}+\left\|u^{\prime}\right\|_{L^{2}\left(0, T ; H^{-1}(U)\right)}\right)
$$

with the constant $C$ depending only on $T$.

Assume that $\Omega$ is bounded and its boundary $\partial \Omega$ is smooth. Take $m$ to be a non-negative integer. Suppose $u \in L^{2}\left(0, T ; H^{m+2}(\Omega)\right)$ with $u^{\prime} \in L^{2}\left(0, T ; H^{m}(\Omega)\right)$. Then

- $u \in C\left(0, T ; H^{m+1}(\Omega)\right)$.

- We have the estimate

$$
\max _{0 \leq t \leq T}\|u(t)\|_{H^{m+1}(\Omega)} \leq C\left(\|u\|_{L^{2}\left(0, T ; H^{m+2}(\Omega)\right)}+\left\|u^{\prime}\right\|_{L^{2}\left(0, T ; H^{m}(\Omega)\right)}\right)
$$

with the constant $C$ depending only on $T, U$ and $m$.

\subsection{Ill-Posed and Inverse Problems}

In the beginning of the last century, the French mathematician J. Hadamard introduced the concept of well-posedness, see [7] and [1].

Definition 2.2.1. A mathematical problem is called well-posed in the sense of J. Hadamard, if the following conditions are satisfied:

1. A solution to the problem exists.

2. The solution is unique.

3. The solution depends continuously on the data of the problem. The third condition is to ensure that small errors in the data will cause small errors in the solution.

If one of the conditions above is not fulfilled, then the problem is called ill-posed.

Hadamard gave an example of an ill-posed problem, namely the Cauchy problem for the Laplace equation, where a harmonic function is to be found given its function values and normal-derivative on a part of the boundaries of the solution domain.

Many interesting and important problems in science and technology lead to inverse problems; that is to determine an unknown functions or to estimate parameters from over-specified data. It turns 
out that inverse problems are ill-posed in the sense that noise in measurement data may lead to significant misinterpretations of the solution. The ill-posedness can be handled either by incorporating a-priori information via the use of transformations, which stabilizes the problem, or by using appropriate numerical methods, called regularization techniques.

Actually, inverse and ill-posed problems arise in various areas of science and technology, for instance in mathematical biology, computer vision, image processing, meteorologi, geophysics, spectroscopy, optics, nuclear physics, and control theory. In the field of traditional mathematics, these problems can be found in complex analysis, functional analysis, differential equations, and in linear algebra.

The theory and methods for solving ill-posed and inverse problems was developed by, among others, A. N. Tikhonov [8-10], F. John [11], M. M. Lavrent'ev [12, 13], and J. L. Lions [14].

Let us give an example of an inverse problem being ill-posed, in the field of mathematical biology.

Example 2.2.2. The reaction-diffusion equation is a well-established model to describe the growth of brain tumours. Let us consider the one-dimensional final-value problem for the reaction-diffusion equation with Neumann boundary conditions:

$$
\begin{cases}\partial_{t} u-D \partial_{x}^{2} u-\rho u=0, & x \in(0, \pi), t \in(0, T), \\ u(x, T)=\psi(x), & 0 \leq x \leq \pi \\ \partial_{x} u(0, t)=\partial_{x} u(\pi, t)=0, & 0 \leq t \leq T,\end{cases}
$$

where $u(x, t)$ is the cell density at the time $t$ at the point $x$. We assume that the final cell density $\psi(x)$ at time $t=T$ is a given function in $L^{2}(0, \pi)$. Without loss of generality, we can assume that the diffusion coefficient $D$ and the reaction coefficient $\rho$, which are both positive and fixed, satisfies the condition $D=\rho=1$. Solving problem (2.1) backwards in time, we obtain the initial cell density $u(x, 0)$ at time $t=0$.

The solution can be obtained by separation of variables in the form

$$
u(x, t)=\sum_{n=1}^{\infty} e^{\left(n^{2}-1\right)(T-t)} \psi_{n} \cos n x,
$$

where $\psi_{n}$ is the $n$-th Fourier coefficient of $\psi$, that is

$$
\psi_{n}=\frac{2}{\pi} \int_{0}^{\pi} \psi(\xi) \cos n \xi d \xi, \quad n=1,2, \ldots
$$


Let $\varphi$ be the initial data: $\varphi(x)=u(x, 0)$. Then, by Parseval's equality, we have

$$
\|\varphi\|_{2}^{2}=\frac{\pi}{2} \sum_{n=1}^{\infty} e^{2\left(n^{2}-1\right) T} \psi_{n}^{2} .
$$

It is obvious that even if $\psi$ is such that the right-hand is finite, small perturbations in $\psi$ do not necessarily cause small perturbations in $\varphi$. This shows that the backward reaction-diffusion equation is ill-posed.

Another way of seeing that the backward reaction-diffusion equation is ill-posed is the following.

Let $\varphi \in L^{2}(0, \pi)$ and let $\varphi_{n}$ be the $n$-th Fourier coefficient of $\varphi$. The solution to the reaction-diffusion equation with the initial condition $u(x, 0)=\varphi(x)$ is given by

$$
\begin{aligned}
u(x, t) & =\sum_{n=1}^{\infty} e^{\left(1-n^{2}\right) t} \varphi_{n} \cos n x \\
& =\int_{0}^{\pi}\left(\frac{2}{\pi} \sum_{n=1}^{\infty} e^{\left(1-n^{2}\right) t} \cos (n x) \cos (n \xi)\right) \varphi(\xi) d \xi .
\end{aligned}
$$

Thus, the function $u$ is a solution to problem (2.1) if $\varphi$ satisfies the following Fredholm integral equation of the first kind:

$$
\int_{0}^{\pi} K(x, \xi, T) \varphi(\xi) d \xi=\psi(x), \quad 0 \leq x \leq \pi,
$$

where

$$
K(x, \xi, T)=\frac{2}{\pi} \sum_{n=1}^{\infty} e^{\left(1-n^{2}\right) T} \cos (n x) \cos (n \xi) .
$$

This is a special case of the equation

$$
B \varphi=\psi,
$$

where

$$
B \varphi(x)=\int_{0}^{\pi} K(x, \xi) \varphi(\xi) d \xi .
$$

It is well-known that $B$ is a compact operator. Hence, $B^{-1}$ is not bounded on $L^{2}(0, \pi)$. Hence, small perturbations in $\psi$ do not necessarily cause small perturbations in $\varphi$. Again, we see that the backward reaction-diffusion equation is ill-posed. 


\subsection{Iterative regularization methods}

There are various approaches to the solution of ill-posed and inverse problems. On the whole, all such approaches can be divided into two large groups. One of the groups comprises of methods based on bringing the problem into the class of well-posed problems in Tikhonov's sense [14]. The other group consists of those methods that use universal regularizing algorithms, which can be obtained with the aid of Tikhonov's parametric functional [10]. One has to remark that it is the latter group of methods that appears to be the most widely used in applications. In the framework of this approach, one can apply various versions of regularizing algorithms, which make use of the reduction of the problem to the solution of integral equations of the first kind. Because of the importance of the problems under consideration, which have applications in many branches of science and technology, and also because of the invariably increasing requirements concerning the accuracy of results, the search for other approaches to the solution of ill-posed and inverse problems is in progress.

Iterative regularization methods have recently found a wide field of applications in practical problems concerning the solution of various ill-posed problems. The methods have a number of unquestionable advantages, which include the simplicity of computational schemes, the similarity of the schemes for problems with linear and non-linear operators, the high accuracy of solutions, etc. The methods have also the important virtue of allowing any restrictions upon the solution that may be essential for the problem (for example, the requirements that the solution be non-negative, monotonic, etc.) to be easily taken into account directly in the scheme of the iterative algorithm.

In their seminal paper [2], V. A. Kozlov and V. G. Maz'ya proposed elegant and computationally feasible iterative procedures for solving ill-posed boundary value problems for PDEs involving selfadjoint operators. A common idea for utilizing these methods is to preserve the equation and in each iteration solve a well-posed problem obtained by changing the boundary conditions.

Later on, Baravdish [3] extended some of the results of Kozlov and Maz'ya [2] to propose iterative regularization methods for illposed boundary value problems for PDEs involving non self-adjoint operators.

Let us recall an iterative regularization method for ill-posed boundary value problems for PDEs studied by Kozlov and Maz'ya and later 
on by Baravdish to solve the inverse problem in Example 2.2.2. This iterative regularization method will be extended in this thesis to the inverse problem of a non-linear reaction-diffusion equation utilizing a reconstruction of initial location of a brain tumour.

We begin by introducing some notation. We denote by $\mathcal{H}$ a separable Hilbert space endowed with the inner product $(\cdot, \cdot)_{\mathcal{H}}$ and the norm $\|\cdot\|_{\mathcal{H}}$.

Definition 2.3.1. Let $A$ be a positive, self-adjoint unbounded operator on $\mathcal{H}$ with the spectral resolution of identity $E$. Denote by $\mathcal{H}^{\alpha}$, $\alpha \leq 0$, the completion of $\mathcal{H}$ in the norm

$$
\|v\|_{\mathcal{H}^{\alpha}}=\left(\int_{0}^{\infty}\left(1+\lambda^{2}\right)^{\alpha} d(E(\lambda) v, v)\right)^{1 / 2} .
$$

For $\alpha>0$, we denote by $\mathcal{H}^{\alpha}$ the set of elements with finite norm (2.2).

Kozlov and Maz'ya have studied, among other ill-posed problems [2], a first order differential equation with a self-adjoint operator coefficient acting on a Hilbert space, which mimics the heat equation, namely an abstract parabolic equation with final boundary condition:

$$
\left\{\begin{array}{l}
\partial_{t} u+A^{2} u=0, \quad 0<t<T \\
u(T)=\psi
\end{array}\right.
$$

where $\psi$ belongs to $\mathcal{H}$. The equation in this problem should be interpreted as

$$
u(t)+A^{2} \int_{0}^{t} u(\tau) d \tau=\varphi, \quad t>0
$$

for some $\varphi \in \mathcal{H}$. Let $T$ be a fixed positive number. We denote by $L^{2}\left(0, T ; \mathcal{H}^{\alpha}\right)$ the set of functions $u=u(t), 0<t<T$, with values in $\mathcal{H}^{\alpha}$ and with finite norm

$$
\|u\|_{L^{2}\left(0, T ; \mathcal{H}^{\alpha}\right)}=\left(\int_{0}^{T}\|u(t)\|_{\mathcal{H}^{\alpha}}^{2} d t\right)^{1 / 2} .
$$

We also define

$$
\mathcal{S}=\left\{u ; u \in L^{2}\left(0, T ; \mathcal{H}^{1}\right), \partial_{t} u \in L^{2}\left(0, T ; \mathcal{H}^{-1}\right)\right\}
$$

with the norm

$$
\|u\|_{\mathcal{S}}=\left(\int_{0}^{T}\left(\|u(t)\|_{\mathcal{H}^{1}}^{2}+\left\|\partial_{t} u(t)\right\|_{\mathcal{H}^{-1}}^{2}\right) d t\right)^{1 / 2} .
$$


By Example 2.2.2 it folllows with $\rho=0$ that the problem (2.3) in the non-abstract case is ill-posed.

The idea of the algorithm by Kozlov and Maz'ya is to solve initial value problems, where the equation is preserved. The following iterative algorithm is proposed. The initial approximation $u_{0}$ is obtained by solving

$$
\left\{\begin{array}{l}
\partial_{t} u_{0}+A^{2} u_{0}=0, \quad 0<t<T \\
u_{0}(0)=\varphi
\end{array}\right.
$$

where $\varphi$ is arbitrary in $\mathcal{H}$. Once we have constructed $u_{k}, k \geq 0$, the next approximation $u_{k+1}$ is the solution to the problem

$$
\begin{cases}\partial_{t} u_{k+1}+A^{2} u_{k+1}=0, & 0<t<T \\ u_{k+1}(0)=u_{k}(0)-\gamma\left(u_{k}(T)-\psi\right) & \end{cases}
$$

Denote by $\sigma(A)$ the spectrum of $A$, i.e., the complement in $\mathbf{C}$ of the set of all $\lambda \in \mathbf{C}$ such that the operator $(A-\lambda I)^{-1}$ exists and is bounded. Put $\lambda_{0}=\inf \{\lambda ; \lambda \in \sigma(A)\}$.

The result below, which is due to Kozlov and Maz'ya [2], shows the convergence of this method.

Theorem 2.3.2. Let $A^{2}$ be a positive self-adjoint operator acting on a Hilbert space $\mathcal{H}$. Let $\varphi_{0} \in \mathcal{H}$ be an arbitrary initial data element for the iterative procedure suggested above and $u_{k}$ be the $k$-th approximate solution. Suppose that $0<\gamma<2 e^{-\lambda_{0}^{2} T}$ and let $u \in \mathcal{S}$ be a solution to problem (2.3). Then we have

$$
\left\|u_{k}-u\right\|_{\mathcal{S}} \rightarrow 0, \quad \text { as } k \rightarrow \infty .
$$

They also give error estimates, rate of convergences and show that this method is optimal.

Since the solution to (2.3) is given by

$$
u(t)=e^{A^{2}(T-t)} \psi,
$$

then the inverse problem of solving the final-value problem in (2.3) can be reduced to the operator equation

$$
B \varphi=\psi,
$$

where $B=e^{-A^{2} T}$ is positive and self-adjoint operator. Moreover, since the operator $B$ is compact, the problem in (2.6) is ill-posed. 
The scheme of approximating the solution in (2.3) can then be written in the following form:

$$
\varphi_{k+1}=\varphi_{k}-\gamma\left(B \psi-\varphi_{k}\right) .
$$

The scheme in (2.6) is called the Landweber method [15] for linear operator equations involving self-adjont positive compact operators.

Baravdish [3] extended the iterative method of Kozlov and Maz'ya to the following final value problem for an abstract first order differential equation:

$$
\left\{\begin{array}{l}
\partial_{t} u+A u=0, \quad 0<t<T, \\
u(T)=\psi,
\end{array}\right.
$$

where $-A$ is a maximal dissipative operator generating a $\left(C_{0}\right)$ contraction semigroup $G(t), t \geq 0$, and $\psi \in \mathcal{H}$. An iterative algorithm was proposed for this problem together with error estimates and showing that this method is optimal.

Let $\varphi_{0} \in \mathcal{H}$ be arbitrary. The initial approximate solution $u_{0}(t)=$ $G(t) \varphi_{0}$ is obtained by solving the Cauchy problem

$$
\left\{\begin{array}{l}
\partial_{t} u_{0}+A u_{0}=0, \quad 0<t<T \\
u_{0}(0)=\varphi_{0}
\end{array}\right.
$$

Suppose that $u_{0}$ has been determined, then we continue to solve the Cauchy problem

$$
\left\{\begin{array}{l}
\partial_{t} u_{k}+A u_{k}=0, \quad 0<t<T \\
u_{k}(0)=\varphi_{k}
\end{array}\right.
$$

for $k=1,2, \ldots$, where

$$
\varphi_{k}=\varphi_{k-1}-\gamma v_{k-1}(T),
$$

the number $\gamma$ satisfies $0<\gamma<2 /\|G(T)\|^{2}$, and

$$
v_{k-1}(t)=G^{*}(t)\left(u_{k}(T)-\psi\right)
$$

is the solution to the problem

$$
\left\{\begin{array}{l}
\partial_{t} v_{k-1}+A^{*} v_{k-1}=0, \quad 0<t<T, \\
v_{k-1}(0)=u_{k-1}(T)-\psi,
\end{array}\right.
$$

where $G^{*}(t), t \geq 0$, is the adjoint operator of $G(t), t \geq 0$, and $A^{*}$ is the adjoint operator of $A$. 
Theorem 2.3.3. Suppose that $u \in L^{2}\left(0, T ; \mathcal{H}_{A}\right)$ is a solution to problem (2.7) and let $\gamma$ satisfy $\gamma<2 /\|G(T)\|^{2}$. Let $\varphi_{0} \in \mathcal{H}$ be an arbitrary initial data element for the iterative procedure suggested above and $u_{k}$ the $k$-th approximate solution. Then

(a) We have

$$
\left\|u_{k}-u\right\|_{L^{2}\left(0, T ; \mathcal{H}_{A}\right)} \rightarrow 0, \quad \text { as } k \rightarrow \infty .
$$

(b) Moreover, if $\varphi_{0}-u(0) \in \mathcal{H}_{A}^{\alpha}$ for some $\alpha>0$, then an upper bound for the rate of convergence of the method is given by

$$
\left\|u_{k}-u\right\|_{L^{2}\left(0, T ; \mathcal{H}_{A}\right)} \leq C(\log k)^{-\alpha / 2} .
$$

It is also shown that the method above is optimal, see [3].

Furthermore, the problem (2.7) can be reduced to the operator equaton

$$
G(T) \psi=\varphi
$$

Thus, it follows by (2.8), (2.9) and (2.10) that it can then be written in the following form:

$$
\varphi_{k+1}=\varphi_{k}-\gamma G^{*}(T)\left(G(T) \varphi_{k}-\psi\right)
$$

The scheme in (2.12) is called the Landweber method [15] for linear operator equations involving non self-adjont positive compact operators.

The methods presented above are regularization methods and are optimal in the following sense.

Definition 2.3.4. A family $R_{k}: \mathcal{Y} \rightarrow \mathcal{X}, k=0,1, \ldots$, of bounded linear operators is called regularizing for equation (2.11) at $\varphi_{0} \in \mathcal{H}$ if there exists a positive $\delta_{0}$ and functions $k(\delta)$ and $\varepsilon(\delta)$ defined on $\left(0, \delta_{0}\right)$ such that $\varepsilon(\delta) \rightarrow 0$, as $\delta \rightarrow 0$, and the inequality $\left\|B \varphi_{0}-\psi\right\| \leq \delta$ implies the estimate $\left\|\varphi_{0}-R_{k(\delta)} \psi\right\| \leq \varepsilon(\delta)$.

Definition 2.3.5. A family $R_{k}: \mathcal{Y} \rightarrow \mathcal{X}, k=0,1, \ldots$, of bounded linear operators regularizes equation (2.11) on a set $\mathcal{M} \subset \mathcal{H}$ if it regularizes equation (2.11) at every element $\varphi_{0} \in \mathcal{M}$ and the functions $k(\delta)$ and $\varepsilon(\delta)$ can be chosen independent of $\varphi_{0} \in \mathcal{M}$.

It is well-known (see Ivanov et al. [16]) that a family $\left\{R_{k}\right\}_{k=0}^{\infty}$ of bounded linear operators regularizes equation (2.11) at $\varphi_{0}$ if $R_{k} \varphi_{0} \rightarrow$ $\varphi_{0}$, as $k \rightarrow \infty$. 
Definition 2.3.6. Let $\delta_{0}$ be a positive number. By a method $P$ of solving problem (2.11) we understand a family of mappings $P_{\delta}: \mathcal{Y} \rightarrow$ $\mathcal{X}, \delta \in\left(0, \delta_{0}\right)$. The accuracy of the method $P$ on a set $\mathcal{M} \subset \mathcal{X}$ is given by the quantity

$$
\Delta(\delta ; \mathcal{M} ; P ; B)=\sup _{\substack{\varphi \in \mathcal{M}, \psi \in \mathcal{Y} \\\|B \varphi-\psi\| \leq \delta}}\left\|P_{\delta} \psi-\varphi\right\|, \quad 0<\delta<\delta_{0} .
$$

A method $P: \mathcal{X} \rightarrow \mathcal{Y}$ is called optimal on $\mathcal{M}$ if there exists a positive constant $C$ such that for all $\delta \in\left(0, \delta_{0}\right)$

$$
\Delta(\delta ; \mathcal{M} ; P ; B) \leq C \inf _{\mathcal{P}} \Delta(\delta ; \mathcal{M} ; \mathcal{P} ; B) .
$$

It is well known that

$$
\inf _{P} \Delta(\delta ; \mathcal{M} ; P ; B) \geq \frac{1}{2} \omega(2 \delta ; M ; B),
$$

where

$\omega(\tau ; \mathcal{M} ; B)=\sup \left\{\left\|\varphi_{1}-\varphi_{2}\right\|_{\mathcal{X}}: \varphi_{1}, \varphi_{2} \in \mathcal{M}\right.$ and $\left.\left\|B \varphi_{1}-B \varphi_{2}\right\|_{\mathcal{Y}} \leq \tau\right\}$, see Ivanov et al. [16].

In this thesis, we will extend the iterative regularization method to a final value for a non-linear PDE

$$
\left\{\begin{array}{l}
\partial_{t} u+A u=0, \quad 0<t<T, \\
u(T)=\psi,
\end{array}\right.
$$

where $A=-\operatorname{div}(D \nabla u)-f(u)$. The problem (2.15) can be reduced to the non-linear operator equation

$$
F(\varphi)=\psi
$$

Hanke et al. [17] studied a convergence analysis of the Landweber method for non-linear operator equations (2.16). Let $F: \mathcal{D}(F) \rightarrow \mathcal{Y}$ with domain $\mathcal{D}(F) \subset \mathcal{X}$ and $\mathcal{X}, \mathcal{Y}$ Hilbert spaces with inner products $(\cdot, \cdot)$ and norms $\|\cdot\|$. The approximate data is denoted $\psi^{\delta}$ and satisfies

$$
\left\|\psi^{\delta}-\psi\right\| \leq \delta
$$

The appropriate fixed point equation for non-linear problems is given by

$$
\varphi=\Phi(\varphi):=\varphi-F^{\prime}(\varphi)^{*}(F(\varphi)-\psi)
$$


where the non-linear operator $F$ is differentiable, $F^{\prime}$ is a locally uniformly bounded Fréchet derivative of $F$ and $F^{* *}$ its adjoint. Based on this, the non-linear Landweber iteration is defined via

$$
\varphi_{k+1}=\varphi_{k}-F^{\prime}\left(\varphi_{k}\right)^{*}\left(F\left(\varphi_{k}\right)-\psi\right), \quad k=0,1,2, \ldots
$$

with $\varphi_{0}$ an initial guess which may incorporate a priori knowledge of an exact solution $\varphi^{*}$.

For well-posed problems, convergence of iterative schemes is proven by fixed point arguments using the operator contraction properties so the convergence theory is generalized for nonexpansive operators $\Phi$ :

$$
\|\Phi(\varphi)-\Phi(\tilde{\varphi})\| \leq\|\varphi-\tilde{\varphi}\|, \quad \varphi, \tilde{\varphi} \in \mathcal{D}(\Phi) .
$$

However, for ill-posed problems the operator $\Phi$ is in general no contraction. For example, if $F$ is a compact operator with a second order Fréchet derivative, and if $X$ is infinite dimensional, then $\lambda=1$ belongs to spectrum of $\Phi^{\prime}\left(\varphi^{*}\right)$. Therefore, the non expansivity of $\Phi$ is replaced by the following local property in a ball $B_{r}\left(\varphi_{0}\right)$ of radius $r$ around $\varphi_{0}$ that guarantees at least local convergence of the iteration method:

$$
\left\|F(\varphi)-F(\tilde{\varphi})-F^{\prime}(\varphi)(\varphi-\tilde{\varphi})\right\| \leq \eta\|F(\varphi)-F(\tilde{x})\|,
$$

where $\eta<1 / 2$ and $\varphi, \tilde{\varphi} \in B_{r}\left(\varphi_{0}\right) \subset \mathcal{D}(F)$.

Proposition 2.3.7. If (2.19) holds and if $\varphi^{*}$ is a solution of (2.16) in $B_{r}\left(\varphi_{0}\right)$, then any other solution $\tilde{\varphi}^{*}$ in $B_{r}\left(\varphi_{0}\right)$ fulfils

$$
\varphi^{*}-\tilde{\varphi^{*}} \in N\left(F^{\prime}\left(\varphi^{*}\right)\right)
$$

and vice versa with $N(\cdot)$ denoting the null space of an operator.

As in the linear case, the Landweber iteration can only converge if (2.16) is properly scaled, therefore it is assumed that

$$
\left\|F^{\prime}(\varphi)\right\| \leq 1, \quad \varphi \in B_{r}\left(\varphi_{0}\right) .
$$

Proposition 2.3.8. Assume that:

- $\varphi^{*}$ is solution of $(2.16)$ in $B_{\frac{r}{2}}\left(\varphi_{0}\right)$

- $\psi^{\delta}$ is perturbed data satisfying (2.17) 
- $k^{*}$ is a termination index of the iteration according to the following stopping rule for $y^{\delta}$ :

$$
\left\|\psi^{\delta}-F\left(\varphi_{k^{*}}^{\delta}\right)\right\| \leq \tau \delta \leq\left\|\psi^{\delta}-F\left(\varphi_{k}^{\delta}\right)\right\|, \quad 0 \leq k \leq k^{*},
$$

with $\tau$ positive number depending on $\eta$ of (2.19):

$$
\tau>2 \frac{1+\eta}{1-2 \eta}>2
$$

If (2.19) and (2.20) hold, then

$$
\left\|\varphi^{*}-\varphi_{k+1}^{\delta}\right\| \leq\left\|\varphi^{*}-\varphi_{k}^{\delta}\right\|, \quad 0 \leq k \leq k^{*}
$$

and if $\delta=0$,

$$
\sum_{k=0}^{\infty}\left\|\psi-F\left(\varphi_{k}\right)\right\|^{2}<\infty
$$

if $\delta \neq 0$, then one can show that

$$
\sum_{k=0}^{k^{*}-1}\left\|\psi^{\delta}-F\left(\varphi_{k}^{\delta}\right)\right\|^{2} \leq \frac{\tau}{(1-2 \eta) \tau-2(1+\eta)}\left\|\varphi^{*}-\varphi_{0}\right\|^{2} .
$$

The inequality 2.24 implies that, if Landweber iteration is run with precise data $\psi=\psi^{\delta}$, then the residual norms of the iterates tend to 0 as $k \rightarrow \infty$. That is, if the iteration converges, then the limit is necessarily a solution of $F(\varphi)=\psi$. The next theorem state this convergence.

Theorem 2.3.9. If (2.19) and (2.20) are satisfied and if (2.16) is solvable in $B_{\frac{r}{2}}\left(\varphi_{0}\right)$, then $\varphi_{k}$ converges to a solution $\varphi^{*} \in B_{\frac{r}{2}}\left(\varphi_{0}\right)$ of (2.16). If $\varphi^{\times}$is the unique solution of minimal distance to $\varphi_{0}$ and if in addition $N\left(F^{\prime}\left(\varphi^{\times}\right)\right) \subset N\left(F^{\prime}(\varphi)\right)$ for all $\varphi \in B_{r}\left(\varphi_{0}\right)$, then $\varphi_{k}$ converges to $\varphi^{\times}$.

In case of perturbed data, it follows from (2.25) that the discrepancy principle (2.21) with $\tau$ as in (2.22) determines a well defined stopping index $k^{*}<\infty$. The next theorem states that this stopping rule renders the Landweber iteration a regularization method.

Theorem 2.3.10. Under assumption of theorem 2.3.9, if $\psi^{\delta}$ fulfils (2.17) and if the perturbed iteration is stopped with $k^{*}(\delta)$ according to the discrepancy principle, then

$$
\varphi_{k^{*}}^{\delta} \rightarrow \varphi^{*}, \quad \delta \rightarrow 0 .
$$


Chapter 2. Mathematical Preliminaries 


\section{Chapter 3}

\section{Mathematical Models for Brain Tumours}

We give a background and collect some results for models of brain tumour growth. This chapter is based mainly on [18].

\subsection{Brain Tumours}

A tumour is an abnormal growth of normal tissue cells due to genetic and epigenetic events. After the tumour grows to a certain size, it starts to actively infiltrate into healthy tissue. This invasion phenomenon is one of the hallmarks of cancer, as described by Hanahan and Weinberg [19]. It is often the last step of a malignant tumour and leads to metastasis and to eventual death of the patient.

Brain tumours cells are widely known to be highly diffusive and infiltrating compared with other types of tumours. They consist of motile cells and are not nearly as dense as others. These cells go out of the original tumour mass, move extremely quickly and wander far away from the tumour centre via natural pathways of higher diffusivity, such as brain white matter fiber tracts. Consequently, the tumour mass has no well-defined edges and it fills up space within the cranial cavity. It can compress, shift, invade and damage healthy brain tissue thereby interfering with normal brain functions causing clinical symptoms such as headache, seizures, neurological and cognitive deficits see Fig. 3.1.

Brain cancer is a common cancer worldwide; in 2012 with nearly 256 thousand new cases, brain cancer contributed to about $1.83 \%$ 


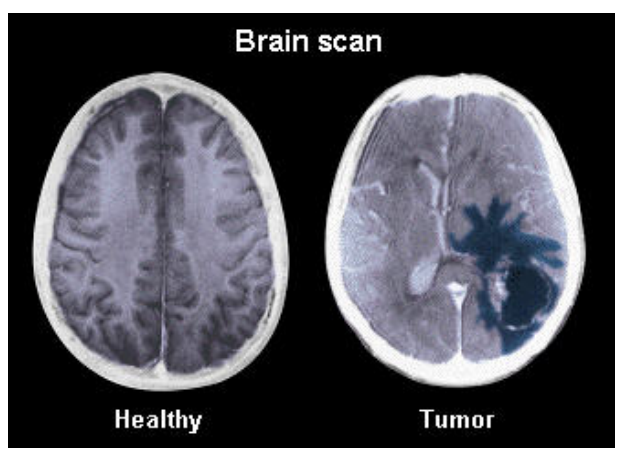

Figure 3.1: A scan of a healthy brain compared to a brain with tumour

of the total number of new cancer cases diagnosed see Fig. 3.2 [20]. Standard treatment includes surgery, radiotherapy and chemotherapy but despite all the therapeutic progress, brain tumours are still rarely fully curable.

Brain tumours are classified based on their origin into primary brain tumours that start in the brain and remain there, and metastatic brain tumours that have spread to the brain from another location in the body. Gliomas are a type of brain tumour that make up about $30 \%$ of all primary and metastatic brain tumours and about $80 \%$ of all malignant brain tumours [21]. The classification is as Grade I through Grade IV according to the World Health Organization (WHO) grading system [22] based on how aggressive the tumour cells of the biopsied tissue appear under a microscope.

Low-Grade Gliomas (LGG) include grade I (astroglial variants that can be cured with complete surgical resection) and grade II (diffusively infiltrating LGG that make surgery difficult with high probability of recurrences: astrocytomas, oligodendrogliomas and mixed gliomas). They are slow growing tumours that look almost normal and infiltrate into normal brain ground tissue. Their progression to a high-grade glioma is almost inevitable [23].

High-Grade Gliomas (HGG) include grade III (Anaplastic astrocytomas) and grade IV (Glioblastomas Multiforma GBM). They look abnormal and are characterized by rapid proliferation, infiltration into large areas. They might also have a necrotic core, form new vascularization to support growth and push the surrounding tissue causing a mass-effect [24]. Fig. 3.3 shows examples of the disease progression. The first row shows a primary glioblastoma growth for a patient: the 


\begin{tabular}{|c|c|c|c|c|c|c|c|c|c|}
\hline \multirow{2}{*}{ Cancer } & \multicolumn{3}{|c|}{ Incidence } & \multicolumn{3}{|c|}{ Mortality } & \multicolumn{3}{|c|}{ 5-year prevalence } \\
\hline & Number & $(\%)$ & ASR (W) & Number & $(\%)$ & $\operatorname{ASR}(W)$ & Number & (\%) & Prop. \\
\hline Lip, oral cavity & 300373 & 2.1 & 4.0 & 145353 & 1.8 & 1.9 & 702149 & 2.2 & 13.5 \\
\hline Nasopharynx & 86691 & 0.6 & 1.2 & 50831 & 0.6 & 0.7 & 228698 & 0.7 & 4.4 \\
\hline Other pharynx & 142387 & 1.0 & 1.9 & 96105 & 1.2 & 1.3 & 309991 & 1.0 & 6.0 \\
\hline Oesophagus & 455784 & 3.2 & 5.9 & 400169 & 4.9 & 5.0 & 464063 & 1.4 & 8.9 \\
\hline Stomach & 951594 & 6.8 & 12.1 & 723073 & 8.8 & 8.9 & 1538127 & 4.7 & 29.6 \\
\hline Colorectum & 1360602 & 9.7 & 17.2 & 693933 & 8.5 & 8.4 & 3543582 & 10.9 & 68.2 \\
\hline Liver & 782451 & 5.6 & 10.1 & 745533 & 9.1 & 9.5 & 633170 & 2.0 & 12.2 \\
\hline Gallbladder & 178101 & 1.3 & 2.2 & 142823 & 1.7 & 1.7 & 205646 & 0.6 & 4.0 \\
\hline Pancreas & 337872 & 2.4 & 4.2 & 330391 & 4.0 & 4.1 & 211544 & 0.7 & 4.1 \\
\hline Larynx & 156877 & 1.1 & 2.1 & 83376 & 1.0 & 1.1 & 441675 & 1.4 & 8.5 \\
\hline Lung & 1824701 & 13.0 & 23.1 & 1589925 & 19.4 & 19.7 & 1893078 & 5.8 & 36.5 \\
\hline Melanoma of skin & 232130 & 1.7 & 3.0 & 55488 & 0.7 & 0.7 & 869754 & 2.7 & 16.8 \\
\hline Kaposi sarcoma & 44247 & 0.3 & 0.6 & 26974 & 0.3 & 0.3 & 80395 & 0.2 & 1.5 \\
\hline Breast & 1671149 & 11.9 & 43.1 & 521907 & 6.4 & 12.9 & 6232108 & 19.2 & 239.9 \\
\hline Cervix uteri & 527624 & 3.8 & 14.0 & 265672 & 3.2 & 6.8 & 1547161 & 4.8 & 59.6 \\
\hline Corpus uteri & 319605 & 2.3 & 8.3 & 76160 & 0.9 & 1.8 & 1216504 & 3.7 & 46.8 \\
\hline Ovary & 238719 & 1.7 & 6.1 & 151917 & 1.9 & 3.8 & 586624 & 1.8 & 22.6 \\
\hline Prostate & 1094916 & 7.8 & 30.7 & 307481 & 3.7 & 7.8 & 3857500 & 11.9 & 148.6 \\
\hline Testis & 55266 & 0.4 & 1.5 & 10351 & 0.1 & 0.3 & 214666 & 0.7 & 8.3 \\
\hline Kidney & 337860 & 2.4 & 4.4 & 143406 & 1.7 & 1.8 & 906746 & 2.8 & 17.5 \\
\hline Bladder & 429793 & 3.1 & 5.3 & 165084 & 2.0 & 1.9 & 1319749 & 4.1 & 25.4 \\
\hline Brain, nervous system & 256213 & 1.8 & 3.4 & 189382 & 2.3 & 2.5 & 342914 & 1.1 & 6.6 \\
\hline Thyroid & 298102 & 2.1 & 4.0 & 39771 & 0.5 & 0.5 & 1206075 & 3.7 & 23.2 \\
\hline Hodgkin lymphoma & 65950 & 0.5 & 0.9 & 25469 & 0.3 & 0.3 & 188538 & 0.6 & 3.6 \\
\hline Non-Hodgkin lymphoma & 385741 & 2.7 & 5.1 & 199670 & 2.4 & 2.5 & 832843 & 2.6 & 16.0 \\
\hline Multiple myeloma & 114251 & 0.8 & 1.5 & 80019 & 1.0 & 1.0 & 229468 & 0.7 & 4.4 \\
\hline Leukaemia & 351965 & 2.5 & 4.7 & 265471 & 3.2 & 3.4 & 500934 & 1.5 & 9.6 \\
\hline All cancers excl. non-melanoma skin cancer & 14067894 & 100.0 & 182.0 & 8201575 & 100.0 & 102.4 & 32455179 & 100.0 & 625.0 \\
\hline
\end{tabular}

Figure 3.2: Estimated cancer incidence, mortality and 5-year prevalence: both sexes worldwide in 2012 (taken from [20])

left MRI revealed a small cortical lesion and the right MRI showed a full-blown glioblastoma with perifocal edema and central necrosis, only 68 days later.

The median survival time is 7.5 years for LGG (grade II) and 18 months for astrocytomas (grade III) with treatments. For GBM, it is 17 weeks without treatment, 30 weeks with radiotherapy and 37 weeks with both surgical resection and radiotherapy.

Besides grading, staging is used to communicate whether and where a tumour has spread in the brain. Staging is typically inferred based on morphological assessment using imaging techniques like Computed Tomography (CT) and Magnetic Resonance Imaging (MRI) [26].

The most common MRI sequences include T1 and T2 weighted images. Gadolinium contrast agent can be administered to the patient in order to acquire $\mathrm{T} 1$ with contrast agent weighted images (T1Gd). T2 weighted FLAIR images (T2-FLAIR) stands for Fluid Attenuation Inversion Recovery T2 images. It is similar to T2 images but with a better contrast due to the attenuation of the signal coming from the cerebro-spinal fluid (CSF). These sequences are preferably performed in at least 2-orthogonal planes or obtained with 
Chapter 3. Mathematical Models for Brain Tumours

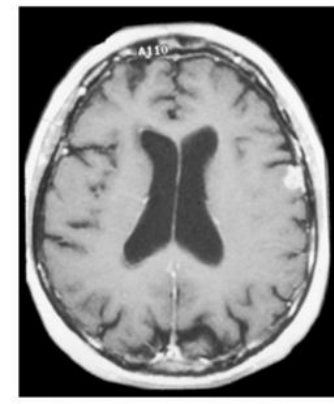

Small cortical lesion

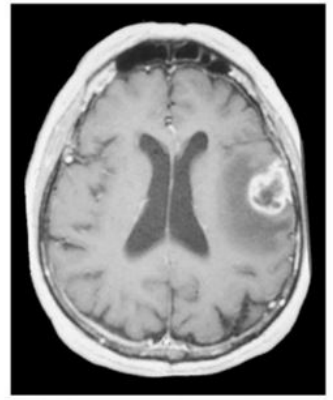

Primary glioblastoma

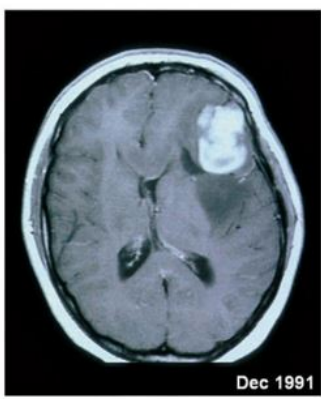

Secondary glioblastoma (WHO grade IV)

Figure 3.3: Progression of brain cancer growth for two patients: in 68 days and in 5 years $([25])$ 
a 3-dimensional (3D) sequence that is reformatted into orthogonal planes (i.e., 3D-T2 FLAIR) [27].

The usual delineated structures of the tumour are the necrotic core, the proliferative rim and the edema. The necrotic core and the proliferative rim are visible on the T1Gd MRI. On the T2 FLAIR, one can delineate the edema of the tumour.

Diffusion Weighted Imaging (DWI) uses the diffusion of water molecules in tissues to generate contrast in MR images and can be used to calculate the Average Diffusion Coefficient (ADC). A special kind of DWI, Diffusion-tensor imaging (DTI), has been used extensively to map white matter tractography in the brain. It involves more directions of interrogation than standard DWI but provides additional parameters and abilities over DWI. DTI provides information on anisotropic diffusion characterized by eigenvectors (direction) and eigenvalues (magnitude), which can be used to derive numerous parameters. The Fractional Anisotropy (FA) derived from DTI is a measure of the directional nature of water diffusivity. It is often anticorrelated to the ADC: high ADC values result in low FA values.

Although MR is the main diagnostic tool for diseases of the central nervous system, Computational Tomography (CT) is still a valuable modality in the imaging of brain tumours (CT involves X-rays which MR does not). CT is superior in detecting calcification, hemorrhage, and in evaluating bone changes related to a tumour [28].

Finally, advanced imaging techniques can also be used such as perfusion images which reveal tumour and tissue vascularity and MR spectroscopy which reveals the tumour metabolic profile.

\subsection{Reaction-Diffusion Models}

The choice of a model is largely guided by the available data. In fact, observations at the microscopic (histological) scale are used to design very detailed mathematical tumour growth models [29]. These proposed theoretical models take the form of ordinary differential equations (ODEs) involving only the density of cells in the tumour:

$$
\frac{d u}{d t}=f(u),
$$

where $u(t)>0$ is the tumour cells density at time $t$ and $f \in C^{1}$ is a function describing the cells proliferation rate through general 
frameworks for tumour growth kinetics. Its expression is given by the generalized logistic equation:

$$
f(u)=\rho u^{\alpha} \beta\left(1-u^{\frac{1}{\beta}}\right)^{\gamma}
$$

where $\alpha, \beta, \gamma$ are non-negative real numbers and $\beta>0$.

Standard models for the growth of brain tumours are classified according to the function $f$ and include:

$$
f(u)=\left\{\begin{array}{lll}
\rho u & \alpha=1, \beta=1, \gamma=0 & \text { Exponential } \\
\rho u(1-u) & \alpha=1, \beta=1, \gamma=1 & \text { Logistic } \\
-\rho u \ln (u) & \alpha=1, \beta \rightarrow+\infty, \gamma=1 & \text { Gompertz }
\end{array}\right.
$$

where $\rho>0$ is the proliferation rate.

The simplest growth assumes a linear relationship of the tumour cell density, resulting in exponential growth stating that cellular division obeys a cycle, with doubling time $\frac{\ln (2)}{\rho}$. This renders a biologically accurate description of tumour growth on time scales that are short in comparison to the life expectancy after the initial tumour development [30]. The logistic and gompertz type growth functions are considered to encapsulate the effect of a maximal capacity of tumour cells incorporating cell population decay with respect to a given carrying capacity.

Such models give a better understanding of cancer initiation and progression by describing the tumour growth process at the cellular level and its interaction with the micro-environment (e.g. in [31] Basanta et al. used evolutionary game theory to build a model that allows to understand certain specific aspects of glioma progression such as the emergence of diffusive tumour cell invasion in Low-Grade tumours and in [32] Kansal et al. developed a three-dimensional cellular automaton model of brain tumour Gompertzian growth). However, these models do not take into account the spatial arrangement of the cells at a specific anatomical location or the spatial spread of the cancerous cells.

As a paradigm, at a given spatial location, new tumour cells appear either by division (proliferation), or by moving from a close location (diffusion). Partial differential equations (PDEs) are suited to describe the space and time evolution of cell densities. They are in fact the tool of choice when studying tumour growth and diffusion into surrounding tissue.

A well-established model for brain tumour growth was formulated by Murray in [33] as a conservation equation. Letting $u(x, t)$ be the 
tumour cells density at a spatial position $x$ (pixel or voxel location in the brain region $\Omega$ inside the skull) and at a time $t$, the governing model is:

$$
\frac{\partial u}{\partial t}=\operatorname{div}(\mathbf{J})+f(u),
$$

where div is the divergence operator and $\mathbf{J}$ is the diffusional flux of cells taken according to Fick's law as proportional to the gradient of the cell density, that is $\mathbf{J}=D \nabla u$, with $\nabla$ the gradient operator and $D$ the diffusion coefficient of tumours cells in the brain tissue. This diffusion term $\operatorname{div}(D \nabla u)$ models the "passive diffusion" (random walk of cells, also called Brownian process) of tumours cells. It does not model cell migration (that is active motion of cells), nor invasion (that is destruction of the extra-cellular matrix). The proliferation term $f(u)$ describe the growth of the population of the tumour cells as described above.

Boundary conditions are imposed to prevent cells from diffusing outside the finite space domain (that is the brain tissue), with a pointsource initial condition of the cell density $\varphi(x)=u(x, 0)$ simulating the start of a tumour. The general form of the model is then given by the following reaction diffusion equation:

$$
\left\{\begin{aligned}
\partial_{t} u-\operatorname{div}(D \nabla u)-f(u) & =0, & \text { in } \Omega \times(0, T) \\
u(0) & =\varphi, & \text { in } \Omega \\
D \nabla u \cdot n & =0, & \text { on } \partial \Omega \times(0, T)
\end{aligned}\right.
$$

where $\Omega$ is the brain region inside the skull and $n$ the outward unit normal vector to the boundary $\partial \Omega$.

In early works, the diffusion tensor $D$ was isotropic in a homogeneous brain structure $\Omega$ :

$$
D=d I
$$

where $I$ is the identity matrix and $d>0$ a constant value for the diffusion coefficient. This kind of tensor allow tumour cells to diffuse equally in all directions and all across the brain. Furthermore, the proliferation of tumour cells was assumed to be exponential $f(u)=$ $\rho u$.

Murray et al. in [34], related the velocity $v$ of the tumour growth to the proliferation rate $\rho$ and the diffusion coefficient $d$ such that $v=2 \sqrt{\rho d}$. This work lead the way to studies by Tracqui et al. [35] that gave rise to the first approximations of model parameters $d$ and $\rho$ using CT scans for HGG. 
The effect of therapy has been included in this model using an additional term describing the death of tumour cells due to therapy (e.g. Cruywagen et al. [36] modeled the effects of chemotherapy on two subpopulations of HGG cells resistent and sensitive and Woodward et al. [37] focused on the resection effect on LGG and HGG).

An extension of the model to 3 -dimensions has been done by Burgess et al. in [38] for LGG and HGG.

In later studies, reaction-diffusion models have been used to describe HGG growth in the heterogeneous environment of the brain. In [39], Swanson et al. also assumed that the tumour cells proliferation was exponential but they improve the model by taking into account the brain tissue heterogeneity so the isotropic diffusion tensor $D$ of equation (1) becomes spatially dependent:

$$
D=d(x) I
$$

where $I$ is the identity matrix and the diffusion coefficient is

$$
d(x)=\left\{\begin{array}{cc}
d_{g} & \text { in grey brain tissue } \\
d_{w} & \text { in white brain tissue }
\end{array}\right.
$$

where $d_{w}>>d_{g}>0$ corresponding to the observation that tumour cells diffuse faster on white matter [40].

Swanson et al. in [41] extended the model further to 3-dimensions taking advantage of the information about white and grey matter areas extracted from the BrainWeb anatomical atlas [42],[43]. They showed how the model parameters could be estimated using only in vivo post-contrast T1 weighted and T2 weighted MRI data.

Other extensions to the model have been done, for example, including biomechanical properties of the brain and considering the fact that glioma cells diffuse more easily along the white matter tracts direction, which can be measured using magnetic resonance diffusion tensor imaging (MR-DTI).

In this context, many authors constructed inhomogeneous diffusion tensors $D$ to model the diffusion of tumour cells from the Diffusion Tensor Images (DTI):

$$
D=\mathbf{D}(\mathbf{x}) .
$$

The tensor construction method consisted of using isotropic (spherical) tensors in grey matter and anisotropic (ellipsoid) diffusion in white matter. 
Using a 3D segmented MRI atlas, Clatz et al. in [44] proposed a model coupling between reaction diffusion and the mechanical constitutive equations to simulate the mass effect (brain deformation induced by the tumour invasion) during GBMs growth. In the reaction diffusion equations, they used exponential growth for the tumour cells proliferation to simplify the model and assumed that in white matter the anisotropic diffusion ratio is the same for water molecules and tumour cells:

$$
\mathbf{D}(\mathbf{x})=\left\{\begin{array}{rc}
d_{g} \mathbf{I} & \text { in grey matter } \\
d_{w} \mathbf{D}_{\text {water }} & \text { in white matter }
\end{array}\right.
$$

where $\mathbf{D}_{\text {water }}$ is the water diffusion tensor in the brain measured by the DTI, i.e., the spatial diffusion of water molecules by MRI per voxel. It contains the full diffusion information along six directions.

To increase the tensor anisotropy in white matter without changing its orientation and also favouring the appropriate orientations when fiber crossing occurs, in their proposed LGG growth model [45], Jbabdi et al. have introduced a formulation which takes into account the possible equality of the two largest eigenvalues corresponding to a possible fiber crossing keeping exponential growth for proliferation and isotropic diffusion in grey matter:

$$
\mathbf{D}(\mathbf{x})=\left\{\begin{array}{r}
d_{g} \mathbf{I} \quad \text { in grey matter } \\
\mathbf{V}(\mathbf{x})\left[\operatorname{diag}\left(\alpha e_{1}(x) d_{w}, d_{g}, d_{g}\right)\right] \mathbf{V}(\mathbf{x})^{T} \quad \text { in white matter }
\end{array}\right.
$$

where $\mathbf{V}(\mathbf{x})$ represents the matrix of sorted eigenvectors obtained by decomposing $\mathbf{D}_{\text {water }}(\mathbf{x}) ; e_{1}(x)$ is the normalized largest eigenvalue (between 0 and 1 ) of $\mathbf{D}_{\text {water }}(\mathbf{x})$ and $\alpha$ is a normalization factor such that the highest $e_{1}$ value in the brain becomes 1 . In this framework, the LGG growth model is directly applied to the extracted tensors without any additional registration.

Besides the prediction of brain tumour growth, the image-based reaction-diffusion models discussed above have been recently used for source localization.

In fact, in [46], Hogea et al. used this model to build an Eulerian framework for modelling tumour growth and its subsequent mechanical impact on the surrounding brain tissue known as the masseffect and introduced an adjoint-based, PDE-constrained optimization problem to estimate the initial tumour seed. For the numerical experiments, they only provided the one-dimensional case without including real images, brain geometry or tissue heterogeneity. Recently, 
Gholami et al. in [47], extended this work for LGG by solving the optimization problem with a reduced space Hessian method.

Konukoglu et al. in [48] proposed a different tool to estimate the time elapsed between the emergence of the tumour and its detection. Based on the reaction-diffusion formalism, they deduced the anisotropic Eikonal equation:

$$
\frac{\sqrt{\nabla u \cdot(D \nabla u)}}{\sqrt{\rho} u}=1, \quad u(\partial \Omega)=u_{0},
$$

describing the extents of the tumour starting from the visible tumour contour in the MR image. With this, one can obtain ODEs to solve for the cell density rather than a PDE. Later on, Rekik et al. in [49] also used this method with the Powell minimization algorithm to estimate the tumour source location for LGG.

\subsection{Discretization}

For numerical implementation of the reaction-diffusion model, we consider an evolution equation of the form:

$$
\partial_{t} u=A u+f(u),
$$

where $A$ is a spatially dependent linear differential operator containing derivatives on its diagonal.

The following explicit iterative discretization scheme is used in time due to its simplicity:

$$
u^{i+1}=u^{i}+h A u^{i}+h f\left(u^{i}\right),
$$

where $i=0,1, \ldots$, is the current iteration, $h>0$ is the stepsize and the matrix $A$, also known as the stencil, can be expressed via a sparse representation making memory requirements less demanding.

We then need to approximate also the spatial derivatives present in $A$. We adopt a generic forward Euler discretization strategy using finite differences approximating derivatives in parabolic PDEs [50].

Expanding the divergence term, we have:

$$
\begin{aligned}
\operatorname{div}(D \nabla u) & =\operatorname{div}\left[\left(\begin{array}{llr}
d_{11} & 0 & 0 \\
0 & d_{22} & 0 \\
0 & 0 & d_{33}
\end{array}\right)\left(\begin{array}{l}
\partial_{x} u \\
\partial_{y} u \\
\partial_{z} u
\end{array}\right)\right] \\
& =\partial_{x}\left(d_{11} \partial_{x} u\right)+\partial_{y}\left(d_{22} \partial_{y} u\right)+\partial_{z}\left(d_{33} \partial_{z} u\right),
\end{aligned}
$$


where the subindex of $d$ are the corresponding components in terms of rows and columns of $D$.

The terms in (3.4) involve derivatives up to second order. We approximate these derivatives by averaging the forward, $\partial^{+}$, and backward, $\partial^{-}$, finite difference operators using the following alternating scheme of forward and backward differences for the $x$-, $y$ - and $z$ directions:

$$
\begin{aligned}
& \partial_{x}\left(d_{11} \partial_{x} u\right) \approx \frac{1}{2}\left(\partial_{x}^{+}\left(d_{11} \partial_{x}^{-} u\right)+\partial_{x}^{-}\left(d_{11} \partial_{x}^{+} u\right)\right) \\
& \partial_{y}\left(d_{22} \partial_{y} u\right) \approx \frac{1}{2}\left(\partial_{y}^{+}\left(d_{22} \partial_{y}^{-} u\right)+\partial_{y}^{-}\left(d_{22} \partial_{y}^{+} u\right)\right) \\
& \partial_{z}\left(d_{11} \partial_{z} u\right) \approx \frac{1}{2}\left(\partial_{z}^{+}\left(d_{33} \partial_{z}^{-} u\right)+\partial_{z}^{-}\left(d_{33} \partial_{z}^{+} u\right)\right) .
\end{aligned}
$$

Letting the size of one voxel be 1 (other sizes can easily be adjusted for) given in the $x$ -,$y$ - and $z$-directions, we derive the forward $\partial^{+}$and backward $\partial^{-}$finite difference operators as second order approximations from a third order Taylor series expansion in the $x-, y$ and $z$-directions:

$$
\begin{aligned}
& \partial_{x}^{+} u=u(x+1, y, z)-u(x, y, z) \\
& \partial_{x}^{-} u=u(x, y, z)-u(x-1, y, z) \\
& \partial_{y}^{+} u=u(x, y+1, z)-u(x, y, z) \\
& \partial_{y}^{-} u=u(x, y, z)-u(x, y-1, z) \\
& \partial_{z}^{+} u=u(x, y, z+1)-u(x, y, z) \\
& \partial_{z}^{-} u=u(x, y, z)-u(x, y, z-1)
\end{aligned}
$$

From this, we get the approximations:

$$
\begin{array}{r}
\partial_{x}\left(d_{11} \partial_{x} u\right) \approx \frac{1}{2}\left[\left(d_{11}(x+1, y, z)+d_{11}(x, y, z)\right)(u(x+1, y, z)-u(x, y, z))-\right. \\
\left.\left(d_{11}(x-1, y, z)+d_{11}(x, y, z)\right)(u(x, y, z)-u(x-1, y, z))\right]
\end{array}
$$




$$
\begin{array}{r}
\partial_{y}\left(d_{22} \partial_{y} u\right) \approx \frac{1}{2}\left[\left(d_{22}(x, y+1, z)+d_{22}(x, y, z)\right)(u(x, y+1, z)-u(x, y, z))-\right. \\
\left.\left(d_{22}(x, y-1, z)+d_{22}(x, y, z)\right)(u(x, y, z)-u(x, y-1, z))\right] \\
\partial_{z}\left(d_{33} \partial_{z} u\right) \approx \frac{1}{2}\left[\left(d_{33}(x, y, z+1)+d_{33}(x, y, z)\right)(u(x, y, z+1)-u(x, y, z))-\right. \\
\left.\left(d_{33}(x, y, z-1)+d_{33}(x, y, z)\right)(u(x, y, z)-u(x, y, z-1))\right] .
\end{array}
$$

Since the boundary of $\Omega$ is the (curved) boundary of the brain (union of white and gray matter segments), special care is needed when computing the Neumann boundary condition on irregular grids. We approach this problem by sequentially replicating the boundary voxels in the outward normal direction of $\Omega$. Any inconsistency for diagonal flow vectors have not been observed in the simulations, in fact this straightforward strategy performs remarkably well. 


\section{Bibliography}

1. Hadamard, J. Lectures on the Cauchy Problem in Linear Partial Differential Equations (Dover, New York, 1952).

2. Kozlov, V. A. \& Maz'ya, V. G. Iterative procedures for solving ill-posed boundary value problems that preserve the differential equations. Algebra i Analiz 1. English transl.: Leningrad Mathematics Journal 1 (1991) 1207-1228, 144-170 (1989).

3. Baravdish (Bastay), G. Iterative methods for ill-posed boundary value problems (Linköping University, Department of Mathematics, Linköping, 1995).

4. Åström, F. Variational Tensor-Based Models for Image Diffusion in Non-Linear Domains $\mathrm{PhD}$ thesis (Linköping University Electronic Press, 2015).

5. Evans, L. C. Partial Differential Equations (American Mathematical Society, 2010).

6. Engl, H. W., Hanke, M. \& Neubauer, A. Regularization of inverse problems (Springer Science \& Business Media, 1996).

7. Hadamard, J. Sur les problèmes aux dérivées partielles et leur signification physique. Princeton university bulletin, 49-52 (1902).

8. Tikhonov, A. N. Solution of incorrectly formulated problems and the regularization method. Soviet Math. Dokl. 4, 1035-1038 (1963).

9. Tikhonov, A. N. Regularization of incorrectly posed problems. Soviet Math. Dokl. 4, 1624-1627 (1963).

10. Tikhonov, A. N. \& Arsenin, V. Y. Solutions of ill-posed problems. VH Winston Ėamp; Sons (Washington, DC: John Wiley \&amp; Sons, New York, 1977). 
11. John, F. Numerical solution of the equation of heat conduction for preceding times. Annali di Matematica Pura ed Applicata 40, 129-142 (1955).

12. Lavrent'ev, M. A., Romanov, V. G. \& Vasiliev, V. G. Multidimensional inverse problems for differential equations (1970).

13. Lavrent_ev, M. M., Romanov, V. G. \& Shishatski_, S. P. Illposed problems of mathematical physics and analysis (American Mathematical Soc., 1986).

14. Lattés, R. L. et al. The method of quasireversibility, applications to partial differential equationsreversibility: applications to partial differential equations (1969).

15. Landweber, L. An iteration formula for Fredholm integral equations of the first kind. American journal of mathematics 73, 615-624 (1951).

16. Ivanov, V. K., Vasin, V. V. \& Tanana, V. P. Theory of linear illposed problems and its applications (Walter de Gruyter, 2002).

17. Hanke, M., Neubauer, A. \& Scherzer, O. A convergence analysis of the Landweber iteration for nonlinear ill-posed problems. Numer. Math. 72, 21-37 (1995).

18. Rockne, R. et al. Modeling diffusely invading brain tumors an individualized approach to quantifying glioma evolution and response to therapy. Selected Topics in Cancer Modeling, 1-15 (2008).

19. Hanahan, D. \& Weinberg, R. A. Hallmarks of cancer: the next generation. cell 144, 646-674 (2011).

20. Ferlay, J. et al. Cancer incidence and mortality worldwide: sources, methods and major patterns in GLOBOCAN 2012. International journal of cancer 136, E359-E386 (2015).

21. Goodenberger, M. L. \& Jenkins, R. B. Genetics of adult glioma. Cancer genetics 205, 613-621 (2012).

22. Louis, D. N. et al. The 2016 World Health Organization classification of tumors of the central nervous system: A summary. Acta neuropathologica 131, 803-820 (2016).

23. Kleihues, P., Burger, P. C. \& Scheithauer, B. W. The new WHO classification of brain tumours. Brain pathology 3, 255268 (1993). 
24. DeAngelis, L. M. Brain tumors. New England Journal of Medicine 344, 114-123 (2001).

25. Ohgaki, H., Burger, P. \& Kleihues, P. Definition of primary and secondary glioblastoma-response. Clinical Cancer Research 20, 2013-2013 (2014).

26. Fass, L. Imaging and cancer: a review. Molecular oncology 2, 115-152 (2008).

27. Mabray, M. C., Barajas, R. F. \& Cha, S. Modern brain tumor imaging. Brain tumor research and treatment 3, 8-23 (2015).

28. Drevelegas, A. \& Papanikolaou, N. Imaging modalities in brain tumors (Springer, 2011).

29. Marušić, M., Bajzer, Ž., Freyer, J. \& Vuk-Pavlović, S. Analysis of growth of multicellular tumour spheroids by mathematical models. Cell proliferation 27, 73-94 (1994).

30. Benzekry, S. et al. Classical mathematical models for description and prediction of experimental tumor growth. PLoS Comput Biol 10, e1003800 (2014).

31. Basanta, D., Simon, M., Hatzikirou, H. \& Deutsch, A. Evolutionary game theory elucidates the role of glycolysis in glioma progression and invasion. Cell proliferation 41, 980-987 (2008).

32. Kansal, A., Torquato, S., Harsh, G., Chiocca, E. \& Deisboeck, T. Simulated brain tumor growth dynamics using a threedimensional cellular automaton. Journal of theoretical biology 203, 367-382 (2000).

33. Murray, J. D. Mathematical Biology. II Spatial Models and Biomedical Applications Interdisciplinary Applied Mathematics V. 18 (Springer-Verlag New York Incorporated, 2002).

34. Murray, J. D. Mathematical Biology. I An Introduction Interdisciplinary Applied Mathematics V. 17 (Springer-Verlag New York Incorporated, 2001).

35. Tracqui, P. From passive diffusion to active cellular migration in mathematical models of tumour invasion. Acta biotheoretica 43, 443-464 (1995).

36. Cruywagen, G. C. et al. The modelling of diffusive tumours. Journal of Biological Systems 3, 937-945 (1995). 
37. Woodward, D. et al. A mathematical model of glioma growth: the effect of extent of surgical resection. Cell proliferation 29, 269-288 (1996).

38. Burgess, P. K., Kulesa, P. M., Murray, J. D. \& Alvord, E. C. The interaction of growth rates and diffusion coefficients in a three-dimensional mathematical model of gliomas. Journal of Neuropathology \& Experimental Neurology 56, 704-713 (1997).

39. Swanson, K. R., Alvord, E. \& Murray, J. A quantitative model for differential motility of gliomas in grey and white matter. Cell proliferation 33, 317-329 (2000).

40. Giese, A. et al. Migration of human glioma cells on myelin. Neurosurgery 38, 755-764 (1996).

41. Swanson, K. R., Alvord, E. \& Murray, J. Virtual brain tumours (gliomas) enhance the reality of medical imaging and highlight inadequacies of current therapy. British journal of cancer $\mathbf{8 6}$, 14-18 (2002).

42. Cocosco, C., Kollokian, V., Kwan, R. \& Evans, A. Brainweb: Online interface to a $3 D$ simulated brain database. Neuroimage 5: S425, 1997

43. Collins, D. L. et al. Design and construction of a realistic digital brain phantom. Medical Imaging, IEEE transactions on $\mathbf{1 7}$, 463-468 (1998).

44. Clatz, O. et al. Realistic simulation of the 3-D growth of brain tumors in MR images coupling diffusion with biomechanical deformation. Medical Imaging, IEEE Transactions on 24, 13341346 (2005).

45. Jbabdi, S. et al. Simulation of anisotropic growth of low-grade gliomas using diffusion tensor imaging. Magnetic Resonance in Medicine 54, 616-624 (2005).

46. Hogea, C., Davatzikos, C. \& Biros, G. An image-driven parameter estimation problem for a reaction-diffusion glioma growth model with mass effects. Journal of mathematical biology $\mathbf{5 6}$, 793-825 (2008).

47. Gholami, A., Mang, A. \& Biros, G. An inverse problem formulation for parameter estimation of a reaction-diffusion model of low grade gliomas. Journal of mathematical biology 72, 409-433 (2016). 
48. Konukoglu, E. et al. Image guided personalization of reactiondiffusion type tumor growth models using modified anisotropic eikonal equations. IEEE transactions on medical imaging 29, 77-95 (2010).

49. Rekik, I. et al. Tumor growth parameters estimation and source localization from a unique time point: Application to low-grade gliomas. Computer Vision and Image Understanding 117, 238249 (2013).

50. Nocedal, J. \& Wright, S. Numerical Optimization (Springer New York, 2006). 
Bibliography 


\section{Chapter 4}

\section{Summary of Papers}

\subsection{Paper 1: Source Localization of Reaction- Diffusion Models for Brain Tumors}

Jaroudi, R., Baravdish, G., Aström, F., and Johansson, B. T. (2016, September). Source Localization of Reaction-Diffusion Models for Brain Tumors. In German Conference on Pattern Recognition (pp. 414-425). Springer International Publishing.

Motivated by the importance of the source localization of brain tumours, we propose a mathematically well-founded approach for locating the source (initial state) of density functions evolved within a non linear reaction-diffusion model. The reconstruction of the initial source is an ill-posed inverse problem since the solution is highly unstable with respect to measurement noise. To address this instability problem, we introduce an iterative regularizing procedure based on the non linear Landweber method for the non linear backward parabolic reaction-diffusion model, and applied it for the stable determination of the initial brain tumours cell density. This amounts to solving a sequence of well-posed forward reaction-diffusion problems. Mathematical analysis of the procedure, such as well-posedness of the forward problems used in the iterations, were undertaken. We show numerically that the source of the initial densities of tumours cells are reconstructed well on both $2 \mathrm{D}$ imaging data consisting of simple and complex geometric structures. These initial experiments indicate that it is possible to retrieve the 
initial source in a stable way with the proposed procedure, and with accepted accuracy. Future work includes, for example, other source terms and validation against real data.

\subsection{Paper 2: Numerical simulations in 3- dimensions of reaction-diffusion models for brain tumours growth}

Jaroudi, R., Åström, F., Johansson, B. T., and Baravdish, G. (2017, Mars). Numerical simulations in 3-dimensions of reaction-diffusion models for brain tumours growth. Submitted to International Journal of Computer Mathematics.

We survey well-established models of reaction-diffusion type for studying brain tumours growth by discussing biological background and mathematical properties. We also give a variational formulation that opens the possibility of alternative derivations and modifications of the models. Moreover, we perform full 3-dimensional simulations of the tumours in time on two types of set-ups, the 3d Shepp-Logan phantom and an MRI T1-weighted brain scan from the Internet Brain Segmentation Repository (IBSR), and for both low and high grade glioma. The reaction term is such that we have logistic growth. These simulations are obtained using standard finite difference discretisation of the space and time-derivatives with novel calculations to increase speed and accuracy, generating a simplistic approach that performs well. In fact, simulations show how the tumour deforms when it reaches regions into which it cannot grow further.

\subsection{Paper 3: Numerical reconstruction of brain tumours}

Jaroudi, R., Baravdish, G., Johansson, B. T., and Åström, F. (2017, July). Numerical reconstruction of brain tumours. Submitted to Inverse Problems in Science and Engineering. 
We investigate the reconstruction of brain tumours backwards in time by formulating the problem as finding the initial state of the solution to well-established parabolic reaction-diffusion models for tumour growth, given the tumour at a final instance in time. We then propose the nonlinear Landweber type method for this inverse problem to obtain a stable solution. Some mathematical analysis was undertaken to find the adjoint operator needed and to motivate convergence. Moreover, we give full 3-dimensional simulations of the tumour source localization for two realistic brain models being the 3-dimensional Shepp-Logan phantom and an MRI T1-weighted brain scan. An additional challenge was introduced in that the simulations was undertaken with the data generated with a different set of parameters (including different nonlinearities). The obtained results corroborated well with what could be expected theoretically, and indicate that stable numerical results can be obtained opening for further investigations of more advanced tumour growth models backwards in time. 


\section{Papers}

The papers associated with this thesis have been removed for copyright reasons. For more details about these see:

http:// urn.kb.se/ resolve?urn=urn:nbn:se:liu:diva-141982 